\title{
DIAGNÓSTICO INSTITUCIONAL DEL PROYECTO EDUCATIVO DE CENTRO EN EL DESARROLLO DE LA EDUCACIÓN INTERCULTURAL
}

\author{
Mario Segura Castillo \\ Asesor Nacional de Evaluación. \\ Departamento de Evaluación MEP 2004. \\ Miembro de la Comisión Institucional de Educación en Formación en valores MEP. \\ Recibido 30-VIII-2004 • Aceptado 09-XI-2004
}

\begin{abstract}
Resumen: Con la creciente inmigración en nuestro país, se debe pensar en el desarrollo de políticas estratégicas de educación intercultural para atender los inmigrantes con justicia y dar mejores condiciones de vida a personas que dejan otras regiones del mundo en busca de mejores oportunidades, aparte de fortalecer los derechos humanos desde su base, es decir, desde el sistema educativo.

El PEC es un "instrumento que recoge y comunica la propuesta integral para dirigir y orientar coherentemente los procesos de intervención educativa que se desarrollan en una intervención escolar" (Antúnez, S. 1992, p. 47).

Se presenta a continuación un estudio de las condiciones que presenta un centro educativo de I y II ciclos de la Educación General Básica con base en un diagnóstico institucional que se realizó para determinar cuáles condiciones de dicho centro educativo favorecen y desfavorecen la puesta en práctica del PEC, centrado en la educación intercultural y en la transversalidad, de acuerdo con la opinión de los docentes y los estudiantes de dicho centro educativo.
\end{abstract}

Palabras clave: Proyecto Educativo de Centro, interculturalidad, transversalidad, temas transversales, alteridad, convivencialidad.

\section{Introducción}

Dentro del marco de un mundo global, los grandes problemas que se viven hoy, como lo manifiesta Martín, J. (2000, p. 88), son, entre otros, la defensa de la identidad o nacionalismo, los movimientos migratorios y la presencia de grandes religiones en los conflictos generados en las sociedades multiculturales.

El planeta cada día se ve estremecido por grupos xenofóbicos, conflictos religiosos y grandes masas humanas que viajan hacia otros países en busca de pan y abrigo, dejando atrás hambre y sangrientas guerras. Por ello, se puede afirmar que lo que ha dejado la globalización es una sociedad multicultural en busca de convertirse en intercultural.

Claro está, en la concepción global del mundo, se ha promovido, desde un punto económico y social del individualismo, la competencia desleal y el etnocentrismo, lo que ha ocasionado que la asimilación cultural sea "la transmisión unidireccional en conformidad con los modelos de la sociedad dominante" (Martín, J. 2000:90).

La dificultad se presenta porque no existe un proceso de integración entre dos o más culturas donde, de una forma sincrética, se llega a incorporar y participar 
Abstract: With the growing immigration taking place in Costa Rica, it is necessary to think about the development of strategic policies of intercultural education in order to give both better attention and living conditions to those who leave other regions of the world looking for better opportunities in our country. Besides, these strategies will support human rights from the point of view of the Costa Rican educational system.

The PEC is an "instrument which collects and communicates an integral proposal to direct and guide coherently the processes of educative intervention that developed in a school intervention process."(1992, $p$. 47). A research study is presented on the conditions of an educational center of and Cycles I and II of the Basic General Education. The study is based on an institutional diagnosis that was carried out to determine which conditions at this school favor or not the practice of the PEC. According to the opinions of students and teachers, the PEC focuses on intercultural education and transversely.

Key words: Central education project, cross-culture, transversally, transversal issues, alteration, cohabitation. activamente en la sociedad, bajo el respeto de la propia identidad y autonomía política en forma equitativa ambas culturas.

Para lograr un verdadero proceso de integración, se deberán crear las condiciones comunicativas y transformadoras de ambas culturas. Lo anterior requiere de un replanteamiento jurídico de respeto a las culturas como integrantes de la misma sociedad.

Desde un punto de vista económico, se debe nivelar la integración para llegar a la equidad y a la igualdad del intercambio.

Tanto la educación como los medios de comunicación deberán buscar formas de cogestión donde le den a los inmigrantes una participación plena y activa en procura de la construcción de valores en el fortalecimiento del respeto e igualdad de oportunidad a todos los grupos culturales del entorno escolar y nacional.

Para Ortega, P. y Mínguez, R. (2001, p. 81), la educación intercultural es una tarea que parte del hecho de que nuestra vida, tanto individual como colectiva, está envuelta en conflictos, expuesta a las diferencias y condicionada por diferentes y legítimos modos de pensar y orientarla. Es esta una realidad a la cual la educación no puede dar la espalda sin renunciar al objetivo fundamental: Educar para la vida. Ello exige afrontar conflictos desde una opción clara para el diálogo, el consenso y el respeto para la dignidad del otro. Conlleva dejar que los conflictos afloren, no suprimirlos, sino asumirlos e integrarlos en el proceso educativo. El término intercultural es usado ampliamente a la hora de hablar de las diversas sociedades y pueblos que constituyen la cultura mundial.

Por esta razón se busca estudiar las condiciones que permitirían o bien, que están obstaculizando la puesta en práctica de un Proyecto Educativo de Centro (PEC). Este proyecto permitiría, entonces, relacionar la educación intercultural con el currículo de la Educación General Básica.

A continuación presentamos el estudio de un diagnóstico institucional aplicado para implementar un PEC centrado en 
la educación intercultural y la transversalidad en una institución de "atención prioritaria" de I y II ciclos de la Educación General Básica.

\section{Breve descripción del proyecto}

El proyecto se realizó en una escuela de la Dirección Regional de San José.

La Escuela tiene una población de aproximadamente 850 estudiantes, que proceden en la mayoría de casos de las "Zonas Urbano Marginales" o de "Atención Prioritaria".

Un porcentaje del $40 \%$ de estos niños ingresa al mercado de trabajo una vez que concluye el primer ciclo de la Educación General Básica.

Los problemas más comunes de la comunidad son la desintegración familiar, problemas de drogadicción, económicos y sociales.

La escuela percibe que el padre de familia ya no cifra esperanzas en la educación de sus hijos, sino que ha focalizado su interés en la parte económica que pueda aportar el niño para el sostén de la familia, sin pensar en el costo social que acarrea esta situación.

Un $40 \%$ de los estudiantes son inmigrantes o hijos de inmigrantes procedentes de Nicaragua, El Salvador y Colombia, así como del interior del país, principalmente de Guanacaste.

\subsection{Aspectos teóricos conceptuales del proyecto}

El PEC sintetiza una propuesta de actuación en un centro educativo, explicita principios y convicciones, es decir, señas de identidad; los objetivos que se pretende llevar a cabo con el PEC; y la estructura organizativa que utiliza el mismo para tratar de conseguir sus propias metas.

El PEC es el primer paso teórico en la planificación de la institución educativa, es el eje vertebrador de la Política Educativa del país y, por tanto, se adscribe al humanismo, al ser la referencia básica de toda la vida escolar. Al ser el PEC la propuesta integral porque avanza en el amplio marco de actuación y de toma de decisiones y al hacer referencia a los diferentes ámbitos de gestión que se desarrollan en la institución, transciende los aspectos propiamente curriculares y necesariamente deberá describir las líneas de actuación, marcar las pautas generales, y señalar los caminos viables para la acción educativa de los diversos estamentos de la comunidad escolar.

De acuerdo con Antúnez (1998, p. 2), el PEC está compuesto por diversos apartados que deben atenderse y estar reflejados en dicho documento, como por ejemplo, los siguientes:

- Análisis del contexto (¿Dónde estamos?). Se debe diseñar en función del contexto escolar concreto, único y específico, lo cual implica la evaluación diagnóstica de una serie de elementos. (Preceptos legales, situación socioeconómica y cultural de la zona, y tipología escolar, entre otros).

- $\quad$ Notas de identidad (¿Quiénes somos?). Consiste en la enumeración y explicación de las notas de la identidad. (Análisis del contexto y propósitos que la institución plantea).

- Formulación de objetivos (¿Qué pretendemos?). La puesta en práctica de un proyecto educativo supone diálogo permanente entre el contenido y la práctica educativa diaria.

- $\quad$ Concreción de la estructura (¿De qué medio disponemos?). La estructura de una institución está formada por el conjunto de elementos y por las relaciones que se establecen en los mismos.

De acuerdo con los elementos anteriores, el PEC puede ser centrado necesariamente en la interculturalidad cuando se determina que en la institución educativa conviven personas de diferentes culturas. 
La interculturalidad "se refiere a la conflictiva convivencia de distintas culturas en un mismo entorno. Este entorno es el propio de una de estas culturas que reconocemos como dominantes, en tanto que las otras culturas se encuentran en minoría" (Méndez, M. 2000, p. 1). Mediante el resultado de cuatro elementos, según Martín, J. (2000, p. 89): a), gran parte de nuestras sociedades han llegado a ser multiculturales y lo serán cada vez más; b) cada cultura tiene sus características, dignas de ser respetadas; c) el multiculturalismo es potencialmente una riqueza; y d) para que esa riqueza logre fructificar, es necesario instaurar una comunicación e interacción entre todas las culturas sin borrar la identidad específica de cada una de ellas.

Desde dicho punto de vista, la educación intercultural lo que busca es la inserción justa de determinada cultura al ámbito global promoviendo la equidad de oportunidades en la vida económica y social. De acuerdo con Martín, J. (2000, p. 89): "Lo intercultural no es un objetivo en sí mismo. Es, sobre todo, un instrumento para promover la igualdad de oportunidades y la mejor inserción posible en la vida económica y social".

Por su parte, la Política Educativa Hacia el siglo XXI toma en cuenta los valores fundamentales del humanismo. Estos valores se relacionan con la solidaridad individual, social y con la naturaleza; dignifican la esencia de los seres humanos. Estos valores humanistas llevan un concepto de humanidad integral en una relación de justicia con la diversidad cultural, la biodiversidad biológica y están relacionados con la búsqueda de un estado físico, fisiológico emocional y mental sano.

De acuerdo con el humanismo de la política educativa, la educación costarricense debería de tener como norte los más altos valores del ser humano en una sociedad democrática e intercultural, donde la igualdad y la libertad serían los más altos fines.

En los intentos por alcanzar el humanismo desde la educación intercultural, este proyecto se propone exponer los resultados que se obtuvieron con base en un diagnóstico institucional para determinar las condiciones de un centro educativo específico para la puesta en práctica de un PEC.

Desde el punto de vista de Palos, J. (2000, p. 37): "El acto por parte del profesorado de transversalizar o de envolver unos contenidos disciplinares con otros, que considera que no están presentes y que son fundamentales en la educación de sus alumnos, siempre ha sido un indicador que el currículo y lo que se enseña en la escuela no recogen suficientemente la realidad social en que están inmersos".

En relación con lo anterior, los temas transversales son aquellos determinados por situaciones problemáticas o socialmente relevantes, generados por el modelo de desarrollo actual, que atraviesan o envuelven el análisis de la sociedad, y del currículum en el ámbito educativo, desde una dimensión ética y en toda su complejidad conceptual. En nuestro país el único eje transversal que existe es el que se refiere a los valores y existen además cuatro temas transversales: La vivencia de los derechos humanos para la democracia y la paz; educación para la salud; cultura ambiental para el desarrollo sostenible, educación para la salud y educación integral de la sexualidad.

Desde el punto de vista educativo, el eje transversal y los cuatro temas transversales deben servir como base para crear el andamiaje social e individual para un desarrollo del pensamiento crítico, la toma de decisiones, la resolución de problemas y el procesamiento de la información. El cambio cualitativo que se necesita para que se produzca una educación acorde con la realidad requiere técnicas donde el estudiante razone, piense y critique. También es necesario salir de la posición conductual en la que se encuentra nuestro sistema, pero lo principal es un docente más democrático pues la escuela ya no puede ser un emporio de autoritarismo militar.

$\mathrm{Al}$ respecto, en la construcción de un Proyecto Curricular de Centro (Buxarrais, 
R. et al.: 2000, p. 55) es necesario considerar la convivencialidad. "La entendemos entonces como la capacidad que tenemos todos los seres humanos para poder compartir un espacio con personas diferentes a nosotros". Para poder propiciar espacios educativos para el desarrollo de la convivencialidad desde la educación intercultural se hace necesario que ésta se fundamente en los principios de respeto, igualdad, solidaridad y justicia.

La discusión podría tornarse en cuáles de las manifestaciones culturales son o no aceptadas en el ámbito del humanismo y cuáles de ellas podrían ser puntos de referencia moral en la construcción de la equidad humana. Es claro que existen manifestaciones culturales que contradicen formas de respeto y tolerancia entre los seres humanos por ser de carácter aberrante y contradictorias, las cuales podrían ser superadas y enriquecidas por otras manifestaciones más humanas. Por lo complejo de entender ciertas manifestaciones culturales aberrantes dentro del ámbito humano, existen una serie de posiciones teóricas al respecto y hoy día se discute si entran en la categoría de "culturales".

Lo anterior permitiría construir un PEC basado en la ética de la alteridad, la cual conforma una perspectiva axiológica que se manifiesta, al menos, desde el punto de vista científico, pedagógico y tecnológico y que consiste en ponerse en lugar del otro, para lograr el respeto y entendimiento de puntos de vista diferentes a los propios.

La base de la ética de la alteridad es la reciprocidad y la solidaridad de las personas, sin que se obligue a nadie a compartir las mismas creencias religiosas, morales o filosóficas de los demás. No se habla sólo de tolerancia, sino de pluralismo razonable en el cual se ve un bien para la comunidad (Hoyos, G; 1998, p. 27).

Otro principio que puede enriquecer la ética de la alteridad es lo que hoy día se conoce como la cogestión educativa: supone, entre otros compromisos, orientar la actitud hacia una educación para la igualdad, "sin discriminaciones por razón de género y superadora de mitos y tabúes. Trata de evitar conductas que ponen énfasis en los roles tradicionales asignados al hombre y a la mujer y procura proporcionar al alumnado imágenes diversificadoras y no convencionales de uno y otro género" (Antúnez, S. 1998, p.4).

\subsection{Metodología aplicada en la realización del proyecto}

El presente informe se enmarca dentro del ámbito interpretativo del paradigma cualitativo. Según Wittrock (1989, p. 196), este enfoque es "más inclusivo que muchos otros. Exige ser especialmente cuidadoso y reflexivo para advertir y describir los acontecimientos cotidianos en el escenario de trabajo y para identificar el significado de las acciones de esos acontecimientos desde los diversos puntos de vista de los propios actores".

\subsubsection{El diagnóstico en la implementación del PEC}

Para la interpretación metodológica, a continuación se realiza una breve descripción de las técnicas e instrumentos utilizados durante la etapa del diagnóstico realizado como primer paso en la implementación del proyecto.

Los procedimientos de recolección de la información abarcaron diversas fuentes durante la realización del diagnóstico institucional:

a) Se hicieron entrevistas mediante un cuestionario con cinco preguntas abiertas aplicadas a veinte docentes, para determinar las actividades promovidas desde el PEC para evitar la xenofobia y las prácticas discriminatorias. Dicho cuestionario incluía preguntas referentes a acciones que desde el centro educativo promueven la tolerancia y la educación intercultural. 
b) También se aplicó, a 35 docentes de I y II ciclos de la institución educativa donde se realizó el diagnóstico institucional, un instrumento de diez frases inconclusas que, de acuerdo con Buxarais, R. (1997, p. 107): “...resultan muy útiles para realizar la primera aproximación a un tema. Es una técnica de carácter individual que consiste en presentar una lista más o menos larga de frases inacabadas o preguntas que obligan a definirse".

Lo que se logró diagnosticar con las frases inconclusas fue la opinión de los docentes con respecto a la implementación del PEC desde la educación intercultural.

c) Las frases inconclusas se utilizaron para realizar una triangulación de la información entre las cuatro preguntas abiertas y el compromiso de los docentes con respecto al PEC centrado en la educación intercultural.

d) Además, se elaboró una lista de cotejo relacionada con 30 preguntas cerradas para determinar la viabilidad de la puesta en práctica del PEC y se le aplicó la misma a treinta y cinco docentes.

e) A sesenta estudiantes de II ciclo se les aplicó una escala tipo Likert con tres constructos: aporte cultural de las diferentes etnias, las diferencias culturales y la tolerancia. Cada uno de los constructos contenía ocho ítemes.

f) Para el presente artículo, con base en la información anterior, se realizó un retrato narrativo analítico que, según Wittrock (1989, p. 167), se refiere a la descripción detallada de los acontecimientos del diagnóstico institucional, pero con el análisis y comentarios del observador desde un punto de vista cualitativo y confrontando los resultados con la teoría existente en educación intercultural.

\section{Descripción de los resultados}

\subsection{Referentes conceptuales obtenidos a partir del diagnóstico institucional}

Dentro de los diferentes aspectos teóricos conceptuales que se pueden señalar, después de haber realizado el diagnóstico institucional para determinar las posibilidades del PEC, se encuentran los siguientes:

- $\quad$ Existen muy pocos proyectos relacionados específicamente con la construcción de valores que se encuentren sistematizados en la institución como parte de la educación intercultural.

- La política educativa vigente en el país propone que a nivel de centro educativo se le dé prioridad a la construcción de valores relacionados con la educación intercultural; sin embargo, en el diagnóstico institucional los docentes consideran que no existe tal necesidad.

- Al nivel de currículum oculto, los docentes proponen actividades de intervención en el aula cuando los estudiantes asumen ciertas actitudes "de rechazo al diferente". Entre otras acciones se promueven:

- El respeto a las diferencias étnicas, así como la discusión crítica del papel de los medios de comunicación acerca del racismo y la xenofobia.

- Actividades relacionadas con la educación intercultural. Los docentes aprovechan las experiencias de aula para promoverlas, por ejemplo, se le da la oportunidad a los extranjeros para que describan las características socio culturales de su país y que las diferencien con respecto a las de Costa Rica. 
- Otro aspecto fundamental es que en la institución educativa se promueve la cooperación en el aula entre estudiantes de diferente origen étnico.

- No obstante lo anterior, en la cultura institucional no se ha sistematizado un PEC para que existan evidencias de la puesta en práctica de la educación intercultural, tal y como debería de formularse. De igual manera, los objetivos generales de la institución no son planeados desde la equidad cultural.

- La falta de sistematización del proyecto educativo de centro es tan evidente, que los mismos docentes indican que éste no se relaciona con la educación intercultural.

- Siguiendo con la misma línea, la contextualización de la realidad social comunal no se toma en cuenta en la programación institucional del centro educativo.

- Aunque se hace referencia al consenso entre padres y docentes para la construcción de valores de convivencia, casi no se toman en cuenta estos valores.

- Las características étnicas de los alumnos no se toman en cuenta en una proporción significativa para elaborar el proyecto del centro educativo.

- Los docentes consideran que existen muy pocos comités en el centro educativo para el desarrollo de las dimensiones morales de los estudiantes.

- $\quad$ Por otra parte, es necesario aclarar que cuando se realizaron las entrevistas y los instrumentos de observación en la escuela, se esperaba encontrar las condiciones mínimas para la puesta en práctica de un proyecto educativo de centro, fundamentado en la educación intercultural y que no existiera la necesidad de la elaboración del mismo. Tampoco se tenía previsto que los docentes desconocieran lo referente a los temas transversales propuestos en los programas de estudio. Todo esto es imprevisto porque la Política Educativa vigente considera que una de las primeras fuentes filosóficas es el humanismo. También fue importante darse cuenta de que los docentes tiene un amplio conocimiento de la ética de la alteridad como una forma de construcción de valores en el centro educativo.

\subsection{Resultados obtenidos acerca de lo que piensan los profesores con respecto a la transversalidad}

Para la implementación de la educación intercultural desde la transversalidad del currículo se pudo constatar que los docentes tienen dos necesidades. La primera, conocer y capacitarse para la educación intercultural; la segunda, capacitarse para el trabajo de los temas transversales y, de esta manera, poder implementar un PEC coherente con los objetivos del proyecto.

Para la puesta en práctica de la educación intercultural desde la transversalidad del currículo en el planeamiento didáctico, se pudieron constatar los siguientes aspectos que consideraron los docentes:

- $\quad$ No se seleccionan contenidos relacionados con la equidad cultural para desarrollarlos como temas transversales.

- Tampoco se implementan equipos de trabajo en el centro educativo para la implementación de los temas transversales.

- Con respecto a los temas transversales, los docentes no realizan planeamiento de la equidad cultural al nivel de aula. 
Es importante establecer que no se esperaba que los docentes tuvieran conocimiento de la ética de la alteridad; sin embargo, ellos consideran que se puede desarrollar una propuesta viable en el proyecto de centro para la implementación de la educación intercultural.

Dentro de los imprevistos, como apuntamos arriba, está el desconocimiento del abordaje que deben realizar los docentes con los temas transversales en el PEC y desde el planeamiento didáctico en el aula. A pesar de que todos los programas de estudio hacen referencia a la transversalidad, no existe, por parte de los docentes, claridad para su puesta en práctica.

\subsection{Resultados obtenidos acerca de lo que piensan los estudiantes con respecto del PEC}

De acuerdo con el diagnóstico realizado en la institución educativa, existen condiciones, según los estudiantes, para que se implemente un PEC para la puesta en práctica de la educación intercultural. A continuación se describe lo que piensan los estudiantes:

- $\quad$ Consideran que los inmigrantes nicaragüenses contribuyen con el fortalecimiento de la cultura costarricense.

- En el centro educativo se promueve en las lecciones la igualdad cultural.

- Existe consenso de los estudiantes para opinar que los nicaragüenses son personas dedicadas al trabajo.

- $\quad$ El progreso del país se ve beneficiado por el comercio que practican los habitantes de origen chino.

- $\quad$ Es importante respetar la identidad cultural de las personas de origen africano. Consideran los estudiantes que es importante el aporte de la cultura afrocostarricense en el desarrollo del deporte.

En síntesis, de lo anterior se deriva que los estudiantes extranjeros son de diferente origen étnico, principalmente nicaragüenses y colombianos. En general, el alumnado practica los valores de la convivencia, la tolerancia, el diálogo y el respeto.

\section{Análisis de los resultados}

\subsection{Análisis acerca de la existencia en educación intercultural en el proyecto educativo de centro}

Al no encontrarse sistematizado en el PEC la educación intercultural desde la transversalidad del currículo, surge como producto de este trabajo una propuesta para desarrollar el PEC, de acuerdo con los siguientes elementos de discusión y análisis del profesorado:

- Desde la educación intercultural es importante que los docentes, como parte de su PEC, construyan el principio de igualdad entre las culturas, la cual se trata de un convencimiento moral y sobre el cual se debe dirigir tal construcción de actitudes y valores en los espacios educativos y comunales. La cogestión educativa juega un papel de suma importancia, debido a que la escuela, junto con las demás instituciones, deben promover campañas concisas sobre el verdadero significado de la igualdad en un mundo donde la diversidad es preponderante.

- También es importante resaltar la participación de los padres junto con los hijos en la tolerancia étnica. Los docentes en su gran mayoría no propician dichos espacios.

- El humanismo, propuesto en la Política Educativa costarricense de cara al presente siglo XXI, se propone rescatar el concepto de ser humano, es decir, dignificar a todos los seres humanos sin distinciones genéricas de ninguna naturaleza. También persigue una visión más globalizadora del ser humano. Se debe basar en 
la búsqueda de un equilibrio entre la diversidad cultural étnica de la humanidad y la diversidad biológica del planeta.

- Con base en el humanismo propuesto en la Política Educativa y desde el punto de vista de las habilidades morales, la ética de la alteridad posibilitaría orientarse a la construcción de personas competentes no sólo en su ejercicio profesional sino en su forma de ser y vivir, guiados por criterios de respeto, solidaridad, justicia y comprensión.

- Lo postulado en el párrafo anterior posibilitaría las capacidades de transformación del entorno: permite la formulación de normas y proyectos contextualizados en los que se pongan de manifiesto criterios de valor relacionados con la implicación y el compromiso, debido a que se pudo constatar que la contextualización de la realidad socio comunal no se toma en cuenta en el PEC.

- Desde esta perspectiva, también la ética de la alteridad se vería enriquecida de la educación moral, la cual supone una tarea constructiva, es decir, exige un trabajo de elaboración personal, social y cultural, de acuerdo con los siguientes componentes:

La construcción de la educación moral parte de un doble proceso de adaptación a la sociedad y a sí mismo.

La personalidad moral se caracteriza por la transmisión de aquellos aspectos culturales y de valor que, pese a no estar completamente enraizados en la trama social, consideramos que son horizontes normativos deseables. En este sentido, la educación intercultural es un eje transversal que todo educador consciente podría construir junto con las habilidades morales.

El segundo momento de la construcción de la personalidad moral se caracteriza por la transmisión de aquellos elementos culturales y de valor que, pese a no estar completamente enraizados en la trama social, consideramos que son horizontes normativos deseables. En este sentido, nadie -o casi nadie- quiere prescindir de una guía de valores como la justicia, la libertad, la igualdad o la solidaridad, ni tampoco quiere abandonar el espíritu y las formas democráticas con las que se desea organizar la convivencia; así mismo, nos resistimos a olvidar propuestas morales como la Declaración Universal de los Derechos Humanos. Elementos culturales de esta naturaleza son la base de creencias y hábitos morales deseables que recogemos en el segundo momento de la educación intercultural. (Puig, J. 1998, p. 191).

Lo anterior coincide con Carbonell i, F. (1992, p. 5) cuando afirma que los aprendizajes resultan deficientes, o ni siquiera se realizan, si el clima en que se llevan a término no es facilitador de los mismos; si el alumnado no se siente reconocido y aceptado en su diversidad; si percibe claramente expectativas desfavorables en relación con el éxito escolar por parte de los profesores; si no consigue relacionar sus aprendizajes con los vínculos socioafectivos que le permitan valorarse al sentirse, a su vez, valorado.

\subsection{Análisis acerca de lo que piensan los profesores con respecto a la transversalidad}

- La puesta en práctica de la educación intercultural en la institución implica la transversalización del currículo así como la realización de talleres de educación intercultural con los docentes, estudiantes y padres de familia, acerca de técnicas y métodos en el desarrollo de las habilidades morales que conlleven al diálogo, tolerancia y respeto de las diferentes etnias que intervienen en el proceso educativo.

- Una de las características de la construcción de la personalidad moral es que ella va a depender del tipo de experiencias que la intervención pedagógica del docente construya junto con los estudiantes y los padres de familia.

- Los estudiantes deberán de enfrentarse a situaciones controvertidas o de un conflicto moral para resolverlas, elaborando y reelaborando las diversas situaciones por medio de la experiencia, la sensibilidad y el diálogo. Para ello son muy importantes los 
conocimientos previos de los estudiantes en los cuales se presentan conflictos de valor y sobre ello trabajar las habilidades morales a través de técnicas e instrumentos en el aula.

- Con respecto de la transversalización del planeamiento didáctico, o sea, a nivel propiamente docente, se deriva del estudio realizado, que este es inexistente, por lo tanto, en el PEC se deberán dar las bases para su construcción.

- $\quad$ Para poder implementar este pla-neamiento didáctico, de acuerdo con Palos, J. (2000, p. 45), la metodología, estrategias y actitudes del profesor, deberían tener una coherencia con los principios metodológicos, con la selección de contenidos priorizada y con el sistema de valores propuesto por el colectivo como proyecto de centro. De acuerdo con el diagnóstico realizado en la institución, se determinó que en el aula se promueven aspectos relacionados con la educación intercultural mientras que en el ámbito institucional, como se explicó arriba, dicha coherencia no existe. Por lo tanto, no es en el planeamiento didáctico de los docentes donde se promueven objetivos para regular la convivencia entre estudiantes, sino más bien que es en el aula donde se promueve el tratamiento de valores cotidianamente relacionados con la educación intercultural.

- Una aportación interesante y necesaria sería identificar los problemas didácticos que plantea cada una de estas y otras estrategias para la transversalidad, lo cual no existe en el centro educativo donde se llevó a cabo el estudio.

\subsection{Análisis acerca de lo que piensan los estudiantes con respecto a la puesta en práctica del PEC centrado en la educación intercultural}

De acuerdo con el estudio realizado, se determinó que los estudiantes consideran que el aporte cultural de las diferentes etnias para poder construir un PEC focalizado en la educación intercultural encuentra líneas de acción en la ética de la alteridad fundamentada en la convivencialidad (capacidad que tienen todos los seres humanos para poder compartir un espacio con personas diferentes), de acuerdo con los siguientes aspectos:

- Considerar a los compañeros por la amistad más que por el color de piel.

- Se deberán de propiciar espacios como, por ejemplo, proyectos desarrollados por los propios estudiantes que contribuyen a campañas institucionales donde se respeten a las personas extranjeras tanto dentro de la institución como en la comunidad.

- Los docentes deben promover en las lecciones el aporte cultural de los afrocostarricenses, chinos y nicaragüenses, desde el punto de vista de la convivencialidad y con un tratamiento transversal desde la educación intercultural.

- Eliminar todas las formas discriminatorias que se practican en la institución y realizar campañas de concienciación en las cuales se asuma una actitud crítica del papel de los medios de comunicación como agentes xenofóbicos de la sociedad moderna. 
- Construir en la institución el valor del respeto a los semejantes y el aprecio a los compañeros por la amistad que brindan sin importar las diferencias étnicas.

- $\quad$ Poner en práctica el valor de la sinceridad con sus compañeros, a pesar de que muchas veces no comparten sus opiniones es importante en las relaciones entre los estudiantes.

- Promover el diálogo con las demás personas en la resolución del conflicto.

- Asumir, por parte de los estudiantes la propia posición ante los problemas relevantes en el ámbito de los valores, para evitar toda práctica discriminatoria. Además se debe asumir una actitud de repudio a toda forma de discriminación que se presente en la comunidad.

Todo lo anterior demuestra que a pesar de la falta de planificación del centro educativo estudiado con respecto a la educación intercultural, los estudiantes tienen una serie de nociones muy claras sobre el respeto, la tolerancia, el diálogo y la convivencia, lo cual resulta altamente positivo para la puesta en práctica del PEC.

Por lo tanto, en los estudiantes del centro educativo estudiado, no existen las bases conceptuales del racismo que describe Prats, E. (2001, p. 140), ya que la modernidad ha modificado los conceptos de espacio y tiempo y ha obligado a revisar la noción de identidad, una noción que hasta ahora se vinculaba de manera estática con tres referentes: la relación con los iguales (o los que considero iguales); la relación con los otros (o los que no considero iguales); la relación con los ancestros (una especie de iguales con la diferencia que no pueden opinar).

De acuerdo con tal posición, se debería realizar una revisión de los términos precedentes: la transgresión referencial, como la superación del contextualismo, pero no del contexto; transgresión diacrítica, como la superación del diferencialismo, pero no de la diferencia y la transgresión diacrónica, como la superación del tradicionalismo, pero no de la tradición.

Es importante realizar una breve descripción de las poblaciones que históricamente sufren las consecuencias de la xenofobia en nuestro país, tales como los hermanos nicaragüenses y los afrocostarricenses.

Dentro del diagnóstico que se realizó en el 2000 la Organización Internacional para las Migraciones (MEP-USAID) determinó la población nicaragüense matriculada en el sistema educativo costarricense en la última década del siglo anterior y los problemas que los inmigrantes presentan en el sistema de educación formal.

Dos problemas detectados por las autoridades de educación y maestros son la inestabilidad escolar de los estudiantes, ya que éstos asisten a las clases en función de los ciclos laborales, lo que origina mucha deserción y dificulta la planificación educativa.

Otro problema es la extra edad o presencia de estudiantes con edades muy superiores al promedio de cada nivel (MEP 2000, p. 29).

Los estudiantes nicaragüenses tienen problemas en su incorporación al sistema educativo, por lo que la concienciación de las autoridades educativas, padres de familia y comunidad se hace indispensable en un PEC que tome en cuenta la educación intercultural.

Con respecto a las personas costarricenses de origen africano, en los últimos años se ha aculturalizado parte de las tradiciones y costumbres de los afrocostarricenses; sin embargo, ha existido un reconocimiento nacional hacia la cultura negra principalmente en el ámbito político y deportivo, al celebrar el "Día del Negro", en la participación política, en los deportes, en las cualidades culinarias y en la profesionalización. Sin embargo, a nivel del país existen muchas críticas negativas debido a que consideran dicha celebración como discriminatoria. En la provincia caribeña, se realiza una celebración que conmemora 
la diáspora humillante y aberrante de su continente natal hacia América.

Desde el punto de vista de la personalidad moral, las anteriores afirmaciones de los estudiantes que aceptan la convivencialidad con sus compañeros extranjeros les posibilitan un progresivo conocimiento de sí mismos, la conciencia del yo, la clarificación de la propia manera de ser, sentir y su valoración como un paso de mucha importancia en la educación intercultural en la construcción del concepto de libertad, de acuerdo con Buxarrais, R. et al. (2000, p. 53). Esto mismo está estrechamente relacionado con el desarrollo de la identidad moral, que a su vez depende de la relación dialógica con los otros, ya que modulamos la propia imagen a partir de las representaciones ajenas, de los roles que nos hacen jugar y de las consideraciones sociales de los mismos. Cuando la persona toma conciencia de sus características, actitudes, valores y principios ejerce su libertad como persona en una sociedad intercultural y democrática.

El conocimiento de uno mismo permite asumir una matriz de valores morales sólida y coherente de manera racional y autónoma. A la vez, permite la elaboración de la autoimagen y favorece niveles superiores de autoestima, ya que la persona es consciente de cómo es, de lo que valora, lo que quiere, y se ve capaz de utilizar los mecanismos y habilidades necesarias para construirse a sí misma, actuando en coherencia en un entorno plural.

Sin embargo, un elemento clave para la construcción en el centro educativo de un proyecto curricular que tenga como eje principal la educación intercultural es la alteridad. Según este concepto, es desde la perspectiva propia que se desarrolla la habilidad de ver las cosas desde la perspectiva del otro, se llega a superar el egocentrismo y conseguimos el reconocimiento de la existencia de puntos de vista diferentes al propio, para lo cual primero el individuo lo interpreta a una escala social y luego al ámbito cognitivo. De acuerdo con Buxarrais, R. et al. (2000, p. 55), esa es la capacidad de transformar el entorno, de cambiar la realidad para mejorarla, de pasar a la praxis, a la acción. Los valores de justicia, dignidad y respeto, de colaboración, esperanza y cambio, de solidaridad serían, entre otros, los que la conforman más directamente.

\section{Conclusiones}

El presente análisis obedece a la interacción que se tuvo con los docentes y estudiantes de la escuela de atención prioritaria, a las preguntas abiertas, entrevistas y a los instrumentos de observación que en un primer momento pretendieron diagnosticar el tópico de estudio y los objetivos planteados $\mathrm{y}$, a preguntas cerradas construidas posteriormente. Por lo tanto, como parte del análisis de los resultados, éstos se pueden reformular con respecto a posteriores estudios que pretendan implementar la educación intercultural, por medio de los temas transversales y la ética de la alteridad, en procura de una verdadera puesta en marcha de los postulados humanistas de la Política Educativa vigente en Costa Rica.

Del tratamiento transversal que se lleva a cabo con el PEC, de acuerdo con los procedimientos, técnicas y métodos que permiten la implementación de la educación intercultural con respecto al centro educativo y su PEC, se puede concluir lo siguiente:

- Acerca de los objetivos del centro educativo, estos no se encuentran expresamente contemplados en el planeamiento institucional, ni en el planeamiento didáctico del docente. En otras palabras, el centro educativo no incluye aspectos interculturales a la hora de hacer su PEC. Esto lleva a situaciones tales como, por ejemplo, que el alumnado perteneciente a etnias o nacionalidades diversas a la nuestra debe adaptarse a un currículo establecido que no ha 
tomado en cuenta sus necesidades, carencias, gustos y habilidades.

- Aunque la mayoría de los docentes afirma que la educación moral se encuentra impregnada en el centro educativo, a la hora de concretar esta "impregnación", son pocos los maestros que lo logran. De aquí se concluye que los objetivos tendientes a la educación moral carecen de un planeamiento formal y sin plasmarse en las actividades y proyectos reales que lleguen al alcance de los estudiantes.

- Con respecto a las técnicas, procedimientos y métodos usados en la educación intercultural, básicamente deberán plantearse aquellos valores que se relacionen con la convivencia, el diálogo y la tolerancia con el fin de insistir, de acuerdo con Prats, E. (2001, p. 187), en la dimensión consciente del individuo como instrumento capaz de superar actitudes y conductas discriminatorias.

- En la formulación de un posible PEC, los docentes mencionan estrategias que les permitirían abordar la educación intercultural como:

- Proyectos para la construcción de los valores.

- Construcción de la personalidad desde la ética de la alteridad.

- Actividades transversales para la construcción de la educación intercultural.

- Asesoramiento para maestros sobre temas transversales.

- Actividades de aula donde se promueve el respeto a las diferencias étnicas.

- Discusión crítica del papel de los medios de comunicación.

- Promoción de proyectos de educación moral.

- Participación de padres e hijos en la tolerancia étnica.
- Aprovechamiento de experiencias de aula para promover la educación intercultural.

- Cooperación intercultural en el aula.

Como resultado de la presente investigación, existe una serie de condiciones que se debe superar antes de la implementación del PEC, como la preparación de los docentes, ya que solo un porcentaje bajísimo ha recibido asesoramientos que le permitan aprender sobre temas transversales, educación intercultural y equidad. Partiendo de estos supuestos, se puede afirmar que la preparación de los docentes en este campo es muy deficiente y que realmente, si llegan a incorporar actividades interculturales, es porque éstas surgen al calor de situaciones de aula o lo han aprendido por otras fuentes; pero no por los medios oficiales o por el seguimiento consciente y sistematizado de la Política Educativa vigente.

- La promoción de proyectos para la educación intercultural, así como la construcción de valores requiere de la intervención de profesionales para capacitar a los docentes. Por medio del estudio realizado, se refleja la necesidad que existe en nuestra sociedad de la promoción de dichos proyectos.

- Por otras parte, al no existir un PEC, el estudiante carece de un soporte institucionalizado y bien desarrollado en materia de proyectos de educación intercultural.

- $\quad$ Sin embargo, un punto positivo es que, como ya se señaló antes, los docentes sí promueven la alteridad en sus lecciones por medio del currículo oculto. De igual manera y según el estudio realizado, el docente promueve la cooperación entre estudiantes de diverso origen étnico.

Al respecto coincidimos con Prats, E (2001, p. 187) cuando considera 
necesarios los principios pedagógicos fundamentales: el de subjetivación, disenso y responsabilidad. Estos sirven para activar los procesos educativos y asumen una función de guía o de punto de referencia para la acción cotidiana.

Por lo tanto, una pedagogía que pretenda abordar la educación intercultural en su PEC, deberá tomar en cuenta los tres principios. Ellos son:

El principio de subjetivación. Asume dos premisas fundamentales: primero, el educando es el sujeto de la educación, y no es un simple objeto de estudio; segundo, el educando es la finalidad de la actuación educativa, y el grupo no es más que un instrumento de esta actuación.

El principio de disenso. Asume una premisa: el espacio público se construye sobre el consenso pero, desde el punto de vista de la pluralidad, es totalmente incorrecto perseguir el bien común a cualquier precio.

El principio de responsabilidad. Se asocia con la capacidad del individuo de dar respuesta, de explicar los términos de su compromiso con los otros y con los iguales; pero no sólo cuando las opiniones lanzadas representan el sentir mayoritario, sino también cuando el consenso no ha recogido las inquietudes minoristas, cuando se detectan situaciones que atentan contra la propia dignidad. En suma, el principio de responsabilidad significa despertar en el estudiante la voluntad y el esfuerzo de autorregular su comportamiento en el sentido de armonizar los intereses individuales con los interese colectivos, de modo que llegue a ser capaz de discernir el bien particular del bien común y asumir la cuota de obligaciones individuales que significa la vida en sociedad.

- Se confirma en el estudio que los docentes sí aplican actividades de aula que promueven el respecto, la alteridad y otras dimensiones de la personalidad moral de los estudiantes. Sin embargo, dichas actividades, según lo muestran los resultados del diagnóstico, no han sido planeadas formalmente por el docente (en su plan semanal o mensual). Se desliga asimismo del estudio que el docente no cuenta con preparación institucional para la realización de actividades valóricas que conlleven a la construcción de una sociedad intercultural. Sin embargo, hay resultados que indican que hay docentes que llevan a cabo actividades donde se promueve el respeto intercultural, pero como ya se puntualizó, estas actividades no se dan en forma coordinada ni con el centro educativo ni con los demás docentes del centro, de ahí que surge la necesidad de la implementación de un PEC que se relacione con la ética de la alteridad y con los principios de acción pedagógica citados arriba.

- En el presente estudio se confirmó que los docentes no toman en cuenta en dicho proyecto las diferencias étnicas a las que se enfrentan hoy día nuestros estudiantes, ante las cuales el centro educativo y los educadores deben estar preparados y no reaccionar de manera instintiva, sino en forma planeada y anticipada. Además, son dejados por fuera del proyecto escolar el planteamiento de objetivos $y$, por ende, de actividades de mediación que tomen en cuenta la igualdad entre jóvenes costarricenses y de otras nacionalidades, lo cual conlleva a que tanto docentes como administradores se enfrenten, sin estar preparados a situaciones difíciles.

- Asimismo, se llegó a determinar las características del PEC de la educación intercultural en la construcción de la personalidad moral de los estudiantes, por lo cual lo más importante es darle un nuevo sentido a la ética de la alteridad enriqueciéndola con el concepto de convivencialidad con las acciones pedagógicas de subjetivación, disenso y responsabilidad. 


\section{Replicación}

Al finalizar el proyecto es importante establecer que al igual que en la escuela de "atención prioritaria" donde se llevó a cabo la intervención, en el medio educativo costarricense hace falta abordar el PEC desde la educación intercultural tomando como referencia varias líneas de acción:

- $\quad$ Primero, un diagnóstico institucional que tome en cuenta técnicas de carácter cualitativo, donde los actores educativos puedan expresar con sinceridad las fortalezas y las debilidades que permitan establecer líneas de acción para promover la verdadera vivencia de los valores humanistas hacia la consecución de los derechos humanos desde la ética de la alteridad.

Segundo, es importante para la replicación del proyecto que a nivel nacional se establezca una política más clara con referencia a los temas transversales y que a la vez sirva como un medio que permita a los actores educativos establecer las prioridades nacionales y particulares de cada institución para promover la convivencia, el respeto y la igualdad de las diferentes etnias y nacionalidades que conviven en nuestra sociedad.

Tercero, falta una culturainstitucional que le permita al sistema educativo crear en las instituciones plataformas donde el PEC se lleve a la práctica realmente y no se convierta en documentos que se dejan en un escritorio.

Por lo tanto, considero que este proyecto podría llevarse a cabo en cualquier centro educativo, siempre y cuando se establezca un PEC de antemano, cumpliendo con todos los requisitos señalados en los tres puntos anteriores. Considero que la puesta en práctica de un PEC es una experiencia necesaria para el desarrollo de la educación intercultural, la cual vendría a fortalecer la educación moral y académica, tal y como señalaba Carbonell i, F. (1992, p. 5) supra.
Por otra parte, el proyecto resulta innovador debido a que en Costa Rica no existe una cultura para la implementación del PEC en los centros educativos y lo que se viene haciendo en la realidad son los FODA (Fortalezas, Debilidades y Amenazas de la institución) como un requisito para presentarlo al supervisor de circuito o cuando llegan los asesores de supervisión nacional.

Además, las instituciones educativas de Costa Rica funcionan con planes y proyectos emanados de un nivel centralizado. Desde este punto de vista, las instituciones educativas necesitan más autonomía y democracia en la toma de decisiones.

Una condición que desfavoreció la realización del proyecto fue una huelga nacional de educadores que duró más de un mes y que coincidió con la finalización del mismo; sin embargo, como un aporte a todos los centros educativos del país y con base al presente estudio, se propone un PEC como uno de los bastiones más importantes de la puesta en práctica de la educación intercultural desde la ética de la alteridad.

\section{Referencias bibliográficas}

Antúnez, S. Del proyecto educativo a la programación del aula. Barcelona: Graó. 1992.

Antúnez, S. Fases en el proceso de integración de la educación en valores en la elaboración de proyectos educativos. Barcelona: UBA. 1998.

Buxarrais, R. La formación del profesorado en valores. España: Desclée. 1997.

Buxarrais, R. et al. La evaluación en la educación en valores. España: OEI - Foro Iberoamericano sobre Educación en Valores Montevideo, Uruguay. 2000.

Carbonell i F. "Decálogo para una educación intercultural”. España: Cuadernos de pedagogía. 1992. 
Cortina, A. La Educación y los valores. Madrid: Argentaria S. A. 2000.

Hoyos, G. "Ética comunicativa y educación para la democracia". Educación, valores y democracia. España: OEI. 1998.

Martín, J. Educar para vivir juntos. Madrid: Argentaria S. A. 2000.

Martínez, M. Consideraciones teóricas sobre la educación en valores. San José C. R.: MEP SIMED. 1998.

Méndez, R. Racismo y xenofobia: El conflicto de la interculturalidad. Madrid: Jornadas. 2000.

Ministerio de Educación Pública. Política Educativa Hacia el siglo XXI. San José: MEP. 1995.

Ministerio de Educación Pública-Agencia del desarrollo Internacional Organización Internacional para Migraciones. Am-pliación de la capacitación del sistema educativo costarricense en las comunidades seleccionadas por la migración nicaragüense relacionada con el Huracán Mitch. San José: MEP. 2000.

Ortega, P. y Mínguez, R. Los Valores en la Educación. España: Ariel. 2001.

Palos, J. Transversalidad y temas transversales: reinterpretar y cambiar el currículo. Madrid: OEI-Foro Iberoamericano sobre Educación en Valores Montevideo, Uruguay. 2000.

Puig, J. "Construcción dialógica de la personalidad moral". Educación, valores y democracia. España: OEI. 1998.

Prats, E. Racismo en tiempos de globalización: Una propuesta desde la educación moral. España: Desclée. 2001.

Wittrock, M. La investigación de la enseñanza, II Métodos cualitativos y de observación. España: PAIDOS. 1989. 